\title{
Memory access: The effects of fact-oriented versus problem-oriented acquisition
}

\author{
LEA T. ADAMS, JANE E. KASSERMAN, A. ALISON YEARWOOD, \\ GREG A. PERFETTO, JOHN D. BRANSFORD, and JEFFERY J. FRANKS \\ Vanderbilt University, Nashville, Tennessee
}

\begin{abstract}
This study examined the effects of similarity between the processing of acquisition and the processing of test materials on performance in a problem solving task. Previous work by Perfetto, Bransford, and Franks (1983) demonstrated that uninformed subjects' failure to utilize relevant acquisition information in a later problem solving task is the result of a failure to spontaneously access such information. The present study demonstrated that spontaneous access can be enhanced when both acquisition and test materials are processed in a similar manner, that is, in a problem-oriented manner. Furthermore, the present findings indicate that the processing similarity leading to enhanced access is specific to particular acquisition and test items, rather than a general problem solving set induced at acquisition and the subsequent testing situation. Results are interpreted within a transfer-appropriate processing perspective.
\end{abstract}

A large body of research supports the idea that people's ability to comprehend, remember, and solve problems requires the availability of previously acquired knowledge (e.g., Bransford, Sherwood, Vye, \& Rieser, 1986; Franks, Bransford, \& Auble, 1982). However, this knowledge must be activated to be useful (e.g., see Bransford \& Johnson, 1972, Experiment 2). By the same token, it is inefficient for people to activate all potential knowledge that may be related to a situation; activation must be selective (e.g., Bransford, McCarrell, Franks, \& Nitsch, 1977). Studies of experts in chess, for example, suggest that it would be too cumbersome to explicitly analyze every possible move that might be made given a particular configuration on a chess board. Instead of accessing every possibility, chess masters are able to consider a relatively small subset of moves that are superior to other possible moves (e.g., see Chase \& Simon, 1973; de Groot, 1965).

In recent years, a number of researchers have begun to explore issues involving access to knowledge. A major feature of this work involves the utilization of experimental paradigms that vary the degree to which subjects are explicitly informed about the relationships between their initial learning experiences and subsequent transfer tests. Most studies of learning and memory are based on an informed paradigm in which subjects are told about the relationship between acquisition and test. For example, subjects may be asked to recall the list of words that was presented during acquisition. This approach to the study of memory and retrieval is quite different from that in

This research was supported in part by ARI contract number MDA90384-0218 awarded to Jeffery J. Franks and John D. Bransford. We wish to thank Janet Metcalfe and two anonymous reviewers for their comments. Requests for reprints should be addressed to Jeffery J. Franks, Department of Psychology, Vanderbilt University, 134 Wesley Hall, Nashville, TN 37240. which uninformed tests of access are used. In the latter situation, subjects are exposed to information that is useful for a subsequent task, such as comprehension, learning, or problem solving, but they are not told about the relationship among the tasks. An important question involves the degree to which subjects spontaneously use information even though they are not explicitly informed about its relevance to their current task.

Asch (1969) provided an excellent demonstration of a lack of spontaneous use of important information. He first had subjects study a list of paired associates until they had mastered all of them (all materials were letter-number pairs, such as C-21, F-18, L-34). Following mastery of the first list, subjects were presented with a second list of pairs to learn. Unknown to the subjects, one letternumber pair on the second list (e.g., C-21) was a pair that had been on the first list, that is, the list the subjects had just mastered. Asch was interested in the number of trials it would take to "learn" this old pair compared with the number required to learn the new pairs that occurred on the second list. Results indicated that the old pair (e.g., $\mathrm{C}-21)$ took as many trials to learn as did entirely new pairs (e.g., X-28) if students failed to notice that the old pair was one that they had previously learned. Sixty-three percent of Asch's subjects failed to notice that they had just learned the critical pair.

A number of other researchers have demonstrated that subjects often fail to utilize relevant information (e.g., Gick \& Holyoak, 1980; Perfetto, Bransford, \& Franks, 1983; Reed, Ernst, \& Banerji, 1974; Simon \& Hayes, 1976; Weisberg, DiCamillo, \& Phillips, 1978). Simon and Hayes (1976) noted that students who learned how to solve the Tower of Hanoi problem did not spontaneously realize that it is structurally isomorphic to the "tea ceremony" problem. Similarly, Gick and Holyoak (1980) showed that, unless students are explicitly prompted to do so, they do not spontaneously use information just 
learned about the solution to the fortress problem to solve an analogous problem (Duncker's, 1945, X-irradiation problem) that they face.

The experiments in the present paper are based on a series of studies by Perfetto et al. (1983). They used acquisition materials that were more obviously relevant to the solution of subsequent problems than was true of materials used in other studies of access (e.g., see their discussion of Weisberg et al., 1978). In addition, the materials permitted multiple responses per subject and hence allowed a more fine-grained analysis of costs and benefits of access than was possible in designs that included only a single problem on the transfer test (e.g, Gick \& Holyoak, 1980; Weisberg et al., 1978).

In Perfetto et al.'s (1983) studies, the problems to be solved were "insight" problems, such as the following:

Uriah Fuller, the famous Israeli superpsychic, can tell you the score of any baseball game before the game starts. What is his secret?

A man living in a small town in the U.S. married 20 different women in the same town. All are still living and he has never divorced one of them. Yet he has broken no law. Can you explain?

Most college students have difficulty answering these questions unless provided with hints or clues. Prior to solving the problem, some students were given clue information that was obviously relevant to each problem's solution. Thus, these students first received cues such as "Before it starts, the score of any game is 0 to 0 " and "A minister marries several people each week." The students were then presented with the problems and explicitly prompted to use the clue information (that was now stored in memory) to solve them. Their problem solving performance was excellent. Other students were first presented with the clues and then given the problems, but they were not explicitly prompted to use the clues for problem solution. Their problem solving performance was very poor; in fact, it was no better than that of baseline students who never received any clues.

Perfetto et al.'s (1983) results represent an especially strong demonstration of access failure, because the clues were constructed to be obviously relevant to problem solutions. Indeed, the authors noted that, before conducting the experiment, they expected even the uninformed students to spontaneously access the correct answers because of the obvious relationship between the problems and the clues.

In retrospect, Perfetto et al.'s (1983) findings of access failure are less surprising when viewed from the perspective of transfer-appropriate processing (Bransford, Franks, Morris, \& Stein, 1979). This perspective emphasizes the importance of similarity between how the target information is initially processed and the processes that are invoked during some later testing situation. For example, Morris, Bransford, and Franks (1977) presented subjects with two different incidental orienting tasks. The first task involved deciding whether a target word fit meaningfully into a sentence frame (e.g., "__ has ears: dog"). The second task involved rhyme judgments (e.g., “__ rhymes with log: dog"). Results indicated that semantic processing (i.e., the former orienting task) led to better memory performance on a recognition test. Previous research had also supported these findings (e.g., Craik \& Tulving, 1975). However, when subjects were presented with a test requiring recognition of words that rhymed with the acquisition target words, subjects who had been exposed to the latter acquisition orienting task exhibited superior performance.

Morris et al.'s (1977) work demonstrated that the similarity between acquistion processing and a later transfer situation can affect performance. It seemed possible that the transfer-appropriate processing perspective might aid in clarifying the lack of transfer (i.e., spontaneous access) in Perfetto et al.'s (1983) experiments. That is, although acquisition answers and their corresponding insight problems were quite similar in informational content, the processes required during acquisition and the subsequent problem solving task were very different. In the acquisition task, subjects read a series of fact-oriented sentences and rated them on general truthfulness. In contrast, the problem solving task was exactly that: subjects were presented with a set of problems and attempted to determine the correct solution for each problem. The relative dissimilarity between the types of processes evoked in the two phases of the experiment might have limited subjects' spontaneous access to acquisition information during subsequent problem solving.

The present work was designed to investigate relationships between transfer-appropriate processing and access. If the transfer-appropriate processing perspective is applicable, then greater transfer (i.e., spontaneous access) should be observed if the acquisition and problem solving tasks are designed to evoke more similar processes. Processing similarity in the experiments was varied by manipulating the form of the acquisition sentences while holding the form of the problems constant. The original sentences used by Perfetto et al. (1983) were modified to invoke problem-oriented processing during the acquisition phase. For example, recall that the original form of the acquisition sentence corresponding to the minister problem was "A minister marries several people each week." To induce problem-oriented processing, this sentence was changed to the following form: "It is possible to marry several people each week if one is a minister." Although the differences between the factual and the problem-oriented form of the sentence may seem rather minor, the latter does evoke, at least momentarily, a problem solving orientation, whereas the former does not. The expectation was that the processes evoked by the problem-oriented acquisition clues would resemble those subsequently evoked during problem solving, and this similarity was expected to lead to enhanced spontaneous access. 


\section{EXPERIMENT 1}

\section{Method}

Subjects. The subjects were 63 undergraduate students enrolled in introductory psychology courses at Tennessee Technological University. The students received extra credit in their courses for participation in the experiment.

Materials. The materials consisted of verbal insight problems and a corresponding set of acquisition sentences that were designed to be obvious answers to the problems. Ten insight problems were a subset of those used by Perfetto et al. (1983). These problems were the same for all experimental conditions. An example problem is as follows:

The Reverend Sol Loony announced that on a certain day, at a certain time, he would perform a great miracle. He would walk for twenty minutes on the surface of the Hudson River without sinking into the water. A big crowd gathered to witness the event. The Reverend Sol Loony did exactly what he said he would do. How did he manage to walk on the surface of the river without sinking?

The aquisition sentences were different for each condition. The students in the fact-oriented condition heard 10 sentences that were identical to those used in Perfetto et al.'s (1983) studies. For the problem-oriented pause condition, the form of the sentences was changed so that the sentence consisted of two clauses, separated by a short (approximately 2 -sec) pause. These sentences were designed so that the acquisition sentences took a problem solving form in that a brief problem (i.e., a difficult to comprehend, unrealistic statement) was presented in the first clause and its solution in the second. The informational content was essentially the same as the sentences in the fact-oriented condition. The acquisition sentences in the problem-oriented no-pause condition were identical to those in the pause condition except that there was no deliberate pause betwern the first and second clauses. The pause/no-pause variation was included as a possible manipulation of the degree of problem solving orientation. Examples of the three sentence types are as follows: (1) A person walking on frozen water will not fall through (fact-oriented). (2) A person walking on water will not fall through (pause) if it is frozen (problem-oriented pause). (3) A person walking on water will not fall through if it is frozen (problemoriented no-pause). The complete set of insight problems and acquisition sentences are presented in the Appendix.

Acquisition sentences were presented on audiotape with a 20 -sec delay between each sentence presentation. The first and last sentences on the tape were filler sentences. The form of these sentences was consistent with the form of the sentences for each experimental condition. Additionally, the first three problems presented during the problem solving tasks were filler problems that were unrelated to any acquisition sentence. The filler problems for all conditions were identical to those used by Perfetto et al. (1983).

Procedure. The subjects were randomly assigned to one of three experimental conditions: the fact-oriented condition, the problemoriented pause condition, and the problem-oriented no-pause condition. The subjects were tested in small groups of 7 to 15 subjects. The general procedure was the same for all three conditions. Each condition consisted of an acquisition phase followed by two test trials. Trial 1 consisted of an uninformed problem solving task. This was followed by Trial 2, which consisted of an informed cue recall task, with the problems serving as the retrieval cues and the goal being to retrieve the acquisition information that was relevant to each particular problem. At the beginning of the experiment, the experimenter told the subjects that they were going to be asked to do three different tasks to help finish several in-progress experiments. This cover story was presented so the subjects would not automatically assume that the acquisition sentences were related to subsequent problem solving tasks.

During the acquisition phase, the subjects were asked to listen to a taped presentation of the 12 sentences ( 2 filler sentences and 10 problem-clue sentences). After each sentence, during a 20 -sec pause, the subjects were to rate the sentence on general truthfulness using a 1 to 5 scale $(1=$ true only in a specific instance to 5 = always true $)$.

There was a 4-min interval between the acquisition task and the subsequent Trial 1 , the uninformed problem solving task. During this time, the experimenter collected the rating sheets and set up for the "next experiment." The subjects were then given a booklet containing three filler problems and half of the insight problems. Based on previous work, the 10 problems were divided into two lists of 5 problems each that were matched on difficulty. One of these lists was presented for uninformed problem solving, and these lists were counterbalanced across subjects. The 5 problems were presented in one of seven random orders

The subjects were told not to open their booklets until instructed to do so. They were then told that they would have $40 \mathrm{sec}$ to read each problem and write an answer. They were not to turn to the next problem until the experimenter told them to do so. Aiso, they were not allowed to go back and work on a previous problem. The subjects were asked to write an answer for every problem, even if they did not believe it was correct.

After the uninformed problem solving trial was completed, subjects were presented with Trial 2 , the informed cued recall test. The experimenter informed the subjects that the acquisition sentences provided answers for the problems, and that they would now receive those same problems plus some new problems that could also be answered with the acquisition sentences. The subjects were given another booklet containing the 3 filler problems followed by all 10 of the insight problems presented in one of 14 random orders. The subjects were asked to try to recall the acquisition sentences using the problems as cues. Again, they had 40 sec for each problem and were not to go backward in the booklet or to turn ahead to the next problem until instructed to do so. The subjects were encouraged to try to recall the acquisition sentences as accurately as possible. They were asked to paraphrase only when a sentence could not be recalled verbatim.

\section{Results and Discussion}

Consistent with the terminology of Perfetto et al. (1983), we termed the five problems presented for both uninformed problem solving (Trial 1) and informed cued recall (Trial 2) "old" problems. The five problems presented only during informed cued recall are termed "new" problems. Data from subjects who had seen more than two of the experimental problems prior to the experiment were dropped from the study. Additional subjects were tested to attain 21 subjects in each condition.

The primary data of interest were the mean proportion of correct solutions to the problems (Trial 1) and the mean number of acquisition statements recalled (Trial 2). Means are presented in Table 1. Data for Trials 1 and 2 were analyzed separately.

Consider first the results of Trial 1 , in which uninformed subjects attempted to solve the problems. A oneway, between-groups analysis of variance (ANOVA) revealed a significant main effect for group $[F(2,60)=3.26$, $p<.05]$. Planned orthogonal contrasts indicated that performance in the two problem-oriented conditions-that is, 
Table 1

Mean Percentages and Standard Deviations of Problems Solved in Experiment 1

\begin{tabular}{|c|c|c|c|c|c|c|}
\hline \multirow[b]{3}{*}{ Condition } & & & \multicolumn{4}{|c|}{ Trial 2} \\
\hline & \multicolumn{2}{|c|}{ Trial 1} & \multicolumn{2}{|c|}{ Old } & \multicolumn{2}{|c|}{ New } \\
\hline & $M$ & $S D$ & $M$ & $S D$ & $M$ & $S D$ \\
\hline act-Oriente & 36.1 & 21.9 & 47.6 & 23.5 & 61.9 & 20. \\
\hline Problem-Oriente & 56.2 & 28.7 & 71.4 & 21.9 & 75.2 & 23.0 \\
\hline Problem-Oriented No-Pause & 51.4 & 26.6 & 64.8 & 26.1 & 72.4 & 19.0 \\
\hline
\end{tabular}

pause and no-pause-did not differ $[t(60)=.63]$, whereas the combination of these two conditions produced significantly higher problem solving performance than did the fact-oriented condition $[t(60)=2.53, p<.02]$. Problemoriented processing during acquisition led to enhanced spontaneous access and problem solving as compared with fact-oriented processing during acquisition. Performance by subjects in the problem-oriented conditions did not significantly differ, suggesting that the pause versus no-pause manipulation induced comparable problem-oriented processing.

The data from Trial 2 (informed cued recall using the problems as cues) were analyzed by a 3 (group: factoriented/problem-oriented pause/problem-oriented nopause) $\times 2$ (problem type: old/new) mixed ANOVA. Results revealed a significant main effect for group $[F(2,60)=5.91, p<.01]$. Also, the solution rates for new problems were superior to those for old problems $[F(1,60)=5.86, p<.05]$. The interaction was not significant.

The main effect for group is due to the enhanced problem solving performance in the problem-oriented conditions. This result is not surprising, given the higher solution rates of these two groups in Trial 1 than of the subjects in the fact-oriented condition.

The fact that new-item performance is superior to olditem performance replicates the findings of Perfetto et al. (1983). They argued that the depressed performance for old items on Trial 2 is due to item-specific interference. That is, during uninformed problem solving, subjects often do not spontaneously access the solutions provided during acquisition. Instead, they attempt to elaborate their own solutions to the problems, and these elaborations are generally inadequate. When subjects are again presented with these same problems for cued recall of acquisition solutions, the encoding specificity relationship between the two problem presentations leads subjects to access their previously generated, inadequate elaborations. This inappropriate access interferes with access to the orignal acquisition information.

In contrast to old problems, new problems were presented for the first time during the informed cued recall test. For new problems, no interfering elaborations would have been previously generated; hence memory performance was better on new problems than old problems. These findings replicate those of Perfetto et al. (1983).

\section{EXPERIMENT 2}

Experiment 2 was designed to further investigate the nature of the processes underlying the enhanced spontaneous access found for problem-oriented acquisition. In the previous discussion, the enhancement was attributed to similarity in the processes induced during initial acquisition and subsequent uninformed problem solving. However, the precise nature of this similarity relation cannot be determined solely by the results of Experiment 1 .

One possible explanation for the findings in Experiment 1 is that the subjects who received the problemoriented acquisition statements were more likely to catch on to the purpose of the experiment than were the subjects in the fact-oriented condition. Since the problemoriented acquisition task involved problem solving, and since the Trial 1 problem solving task involved problem solving, the subjects in the problem-oriented conditions were perhaps more likely to suspect that the information provided during acquisition was relevant for the Trial 1 task. We refer to this explanation as the "general set" hypothesis.

An alternate explanation of the greater spontaneous access for problem-oriented acquisition focuses on knowledge-specific aspects of access. For example, if the statement "A person walking on water will not fall through if it is frozen" appeared during acquisition, it could induce problem-oriented processes that are specific to that problem. When essentially the same problem reappears during uninformed problem solving, it could again induce problem solving processing that is specific to that problem. If so, the similarity between such problem. specific processes may be the basis for enhanced spontaneous access.

These alternative hypotheses (i.e., the general set hypothesis and the knowledge-specific hypothesis) can be tested by modifying the design of Experiment 1 so that problem-oriented and fact-oriented acquisition conditions are manipulated within (instead of between) subjects. Suppose that during acquisition subjects receive five problemoriented acquisition items and five fact-oriented acquisition items. Then, during uninformed problem solving, one group of subjects (those in the knowledge-specific condition) is presented with the five problems corresponding to the problem-oriented acquisition items. A second group of subjects (those in the general set condition) receives five problems corresponding to the fact-oriented acquisition items. (Note that subjects in both conditions received problem-oriented acquisition items.) Thus, the groups should be comparable in terms of any general set about the purposes of the experiment. If the increased problem solving performance found in Experiment 1 were due to a general set created by experiencing some problem solving items during acquisition, then both groups should show enhanced uninformed problem solving relative to a third group receiving only fact-oriented items during 
acquisition. In contrast, if problem-specific processes mediate spontaneous access, then the knowledge-specific condition should show greater spontaneous access than the general set condition. This logic forms the basis for the design of Experiment 2.

\section{Method}

Subjects. The subjects were 73 Vanderbilt University undergraduates enrolled in introductory psychology. They received course credit for their participation. The subjects were tested in small groups of 7 to 11 persons.

Materials. The materials (i.e., fact-oriented and problem-oriented acquisition sentences and test problems) were identical to those used in Experiment 1.

Procedure. The procedures were very similar to those of Experiment 1. As in Experiment 1, there was an acquisition phase that was followed by two test trials. Trial 1 was an uninformed problem solving task. Trial 2 again consisted of an informed cued recall test in which the problems served as cues.

Two major design modifications were introduced. First, only factoriented and problem-oriented pause sentences were used in acquisition. The no-pause condition from Experiment 1 was not included. Second, the contrast between fact-oriented and problem-oriented acquisition sentences was manipulated within, rather than between, subjects. The subjects in all conditions received 10 acquisition sentences to be rated for general truthfulness. They were then presented 5 problems for uninformed problem solving (Trial 1). Finally, these 5 old problems and the remaining 5 new problems were randomly intermixed and presented in the informed cued recall test on Trial 2. The subjects participated in one of four experimental conditions: fact-oriented, knowledge-specific, general set A, and general set B. Since the design of this experiment is somewhat complicated, it is presented in outline in Table 2.

The fact-oriented condition is essentially a replication of the factoriented condition in Experiment 1. During acquisition, these subjects were presented with 10 fact-oriented sentences. During Trial 1, uninformed problem solving, they were presented with problems corresponding to 5 of the acquisition sentences, and during Trial 2 , informed cued recall, they were presented with all 10 problems corresponding to the acquisition sentences (i.e., 5 old and 5 new problems). This condition provides a reference level for examining potential enhanced spontaneous access in the problem-oriented conditions.

In the knowledge-specific condition, the subjects received a blocked acquisition list consisting of 5 fact-oriented items followed by 5 problem-oriented items. The 5 problems presented during Trial 1, uninformed problem solving, corresponded to the 5 problem-oriented acquisition items. The acquisition blocking order maximized the potential induction of a general problem solving set immediately prior to uninformed problem solving. The testing was specific to the problem-oriented items. Thus, this condition could potentially benefit from both a general set concerning the nature of the experiment and from knowledge-specific effects. During Trial 2 , informed cued recall, the subjects were presented with all

Table 2

Design of Experiment 2 Involving Fact-Oriented (FO) and Problem-Oriented (PO) Items

\begin{tabular}{lllc}
\hline \multicolumn{1}{c}{ Condition } & Acquisition & Trial 1 & Trial 2 \\
\hline Fact-Oriented & 10 FO & 5 FO & 5 old FO, 5 new FO \\
Knowledge-Specific & 5 FO, 5 PO & 5 PO & 5 old PO, 5 new FO \\
General Set A & 5 FO, 5 PO & 5 FO & 5 old FO, 5 new PO \\
General Set B & 5 PO, 5 FO & 5 FO & 5 old FO, 5 new PO \\
\hline
\end{tabular}

10 problems as cues with 5 (old) problems corresponding to the problem-oriented acquisition items and 5 (new) problems corresponding to the fact-oriented acquisition items.

The subjects in the general set A condition received a blocked acquisition list identical to that of the knowledge-specific condition. However, the 5 problems presented during Trial 1, uninformed problem solving, corresponded to the 5 fact-oriented acquisition items. This condition was similar to the knowledge-specific condition in that a potential general problem solving set was induced immediately prior to uninformed problem solving. However, in this case, the subjects were tested on problems corresponding to the fact-oriented acquisition sentences, not the problem-oriented sentences. Testing on problems corresponding to the fact-oriented acquisition items permitted assessment of general set effects unconfounded by knowledge-specific effects. During Trial 2, informed cued recall, the subjects were presented with all 10 problems as cues with 5 (old) problems corresponding to the fact-oriented acquisition items and 5 (new) problems corresponding to the problemoriented acquisition items.

Finally, the general set B condition was identical to the general set $A$ condition except that the blocking of acquisition items was reversed. That is, the 5 problem-oriented items were followed by 5 fact-oriented items. As in the general set A condition, during Trial 1, uninformed problem solving, the general set B subjects were tested on the 5 problems corresponding to the fact-oriented acquisition items. This condition served as a control for recency effects that might enhance performance in the knowledge-specific condition relative to the general set $\mathrm{A}$ condition. That is, the test problems in the latter condition corresponded to the first 5 items presented during acquisition. In contrast, knowledge-specific subjects were tested on problems corresponding to the last 5 acquisition items. As a recency control, the general set B subjects were also tested on problems corresponding to the last 5 acquisition items. During Trial 2, informed cued recall, the subjects were presented with all 10 problems as cues, with 5 (old) problems corresponding to fact-oriented acquisition items and 5 (new) problems corresponding to problem-oriented acquisition items.

A final point is important for subsequent analyses and discussion. Note that in all conditions, Trial 2, informed cued recall, consisted of 5 old and 5 new problems. However, due to the manipulation of the type of problems received during Trial 1, at Trial 2 the type of new items varied across conditions. In the fact-oriented and knowledge-specific conditions, new items corresponded to factoriented acquisition items. In the general set A and B conditions, new items corresponded to problem-oriented acquisition items.

As in Experiment 1, items were counterbalanced across conditions.

\section{Results and Discussion}

The results are presented in Table 3. As in Experiment 1, the data for Trial 1 (uninformed problem solving) and Trial 2 (informed cued recall) were analyzed separately. The results of primary interest are those of Trial 1. The findings are straightforward. Contrasts indicated that performance of the two general set groups did not differ $[t(69)=.53]$ and that the combination of performance of the two general set groups did not differ from performance of the fact-oriented group $[t(69)=$ $.63]$, but that together these three groups showed significantly worse problem solving performance than the knowledge-specific group $[t(69)=2.85, p<.01]$. These results replicate the Trial 1 results of Experiment 1. (Note 
Table 3

Mean Percentages and Standard Deviations of Problems Solved in Experiment 2

\begin{tabular}{|c|c|c|c|c|c|c|}
\hline \multirow[b]{3}{*}{ Condition } & \multirow{2}{*}{\multicolumn{2}{|c|}{ Trial 1}} & \multicolumn{4}{|c|}{ Trial 2} \\
\hline & & & \multicolumn{2}{|c|}{ Old } & \multicolumn{2}{|c|}{ New } \\
\hline & $M$ & $S D$ & $M$ & $S D$ & $M$ & $S D$ \\
\hline Fact-Oriented & 35.4 & 23.9 & 52.4 & 23.6 & 70.2 & 18.8 \\
\hline Knowledge-Specific & 49.2 & 25.4 & 69.7 & 18.8 & 83.9 & 16.6 \\
\hline General Set A & 28.9 & 21.8 & 49.3 & 28.4 & 74.2 & 24.5 \\
\hline General Set B & 32.6 & 16.5 & 44.2 & 21.7 & 78.7 & 16.2 \\
\hline
\end{tabular}

that the problem-oriented conditions in Experiment 1 were equivalent to the present knowledge-specific condition.)

The pattern of results supports a knowledge-specific interpretation of the findings of Experiments 1 and 2. That is, problem-oriented sentences induce a problem solving set during acquisition. The processes involved in this set are specific to the encodings of particular sentences. There is a high similarity between these processes and the processes invoked in attempting to solve the corresponding problems during uninformed problem solving. In agreement with the transfer-appropriate processing perspective, this similarity leads to transfer. In this case, the transfer involves spontaneous access to the acquisition sentences and, thus, enhanced solution rates for those specific problems.

The present work provides no evidence for problemoriented processes operating at a more general level. If anything, performance in the general set conditions was somewhat lower than that in the fact-oriented conditions. The general set hypothesis was that problem-oriented acquisition sentences would induce subjects to realize the relationship between problem solving on Trial 1 and initial acquisition, because both involved problem solving. The findings of the present experiment provide no support for the hypothesis that a general tendency to catch on to the experiment was responsible for the greater spontaneous access found in the problem-oriented conditions used in Experiments 1 and 2.

The results of the final, informed cued recall test were also straightforward (see Table 3 ). There was a significant main effect for old versus new items $[F(1,65)=37.8$, $p<.001]$, a main effect for conditions $[F(3,65)=4.36$, $p<.01$ ] , and a nonsignificant interaction. This replicates the findings of Experiment 1 and supports the same interpretations. The enhanced solution rates for new, compared with those for old, items demonstrates the itemspecific interference effect originally reported by Perfetto et al. (1983) and replicated in Experiment 1.

The main effect for conditions is essentially due to superior problem solving performance in the knowledgespecific condition. This is what would be expected, given the knowledge-specific condition's superior spontaneous access and problem solving during Trial 1 . The Trial 1 advantage shown by the knowledge-specific condition is maintained on Trial 2.

Finally, the results of Trial 2, informed cued recall, are important for another reason. The pattern of findings allows rejection of a possible alternative interpretation of the results. It was possible that problem-oriented sentences were inherently more memorable than fact-oriented sentences. The superior performance of the problem-oriented conditions in Experiment 1 might have been due to greater memory strength for problem-oriented items and not due to transfer-appropriate processing similarities. The data for the new items can be used to assess this alternative hypothesis. New-item performnce is critical since it is uncontaminated by prior uninformed problem solving.

The new problems in the fact-oriented and knowledgespecific conditions corresponded to fact-oriented acquisition items. The new problems in the general set $A$ and $B$ conditions corresponded to problem-oriented acquisition items. If problem-oriented items resulted in superior memory strength, then the new-item performance in the general set conditions should be better than new-item performance in the fact-oriented and knowledge-specific conditions. As is evident in Table 3, this was not in fact the case. The mean, new-problem solution rates for the former and the latter two conditions were .766 and .770 , respectively.

An alternative, and maybe more precise, comparison involves the knowledge-specific condition and the two general set conditions, since these three conditions are the most equivalent in terms of acquisition conditions. Their acquisition lists all involved a mixed list of fact-oriented and problem-oriented items, whereas the fact-oriented condition involved only fact-oriented items. This comparison also provides no evidence for differential memory for the two types of acquisition items. In fact, recall of new fact-oriented items (in the knowledge-specific condition, $M=.839$ ) is slightly higher than the recall rates of new problem-specific items in either general set A or B conditions ( $M=.742$ and .787 , respectively). Finally, we can consider data from Experiment 1 that might be relevant to the differential memory hypothesis. The comparison involves recall of new fact-oriented items in the fact-oriented condition $(M=.619)$ with recall of new problem-oriented items in the problem-oriented pause and no-pause conditions ( $M=.724$ and .752 , respectively). Although there appears to be some memorial advantage for the problem-specific items, the comparison of the former with the latter conditions was not statistically significant. Also, note that these comparisons are not as precise as those involving the knowledge-specific and general set conditions in Experiment 2 since the acquisition lists were not comparable mixed lists in Experiment 1. Thus, overall, there is little evidence for any differential memorability for fact- versus problem-specific acquisition encoding conditions, and certainly no evidence for memory differences large enough to account for the performance differences observed on Trial 1, uninformed problem solving.

\section{GENERAL DISCUSSION}

Results of the present experiments demonstrate that problem-oriented acquisition can lead to enhanced spontaneous access during later problem solving. The pattern 
of results indicates that this enhancement is specific to particular problems that correspond to particular related acquisition sentences. The findings are interpreted within the perspective of transfer-appropriate processing. That is, problem-oriented processing during acquisition facilitates subsequent, uninformed problem solving only when the latter problems share content and processes that are similar to the acquisition experiences.

These data are especially interesting in light of experiments conducted by Gick and Holyoak (1983). They were able to induce spontaneous access when subjects received two different illustrations of a general solution strategy that could be applied to a transfer problem. Given their design, it is not possible to say whether their findings were due to general set effects or to knowledge-specific effects. Subjects who received two (rather than one) acquisition stories involving problem solving might have been more likely to catch on to the experiment or to assume that there was a connection between acquisition and subsequent problem solving.

In his 1980 article on problem solving and instruction, Simon provided a theoretical framework that is consistent with the results of the present study. His theory is consistent with the general idea of transfer-appropriate processing yet adds precision to this concept. Simon argued that the knowledge that underlies competent performance in any domain is represented as productions rather than as mere facts or propositions. Productions involve condition-action pairs and hence provide information about the critical features of problem situations that make particular actions relevant. For example, it is one thing to know the definitions of mean, median, and mode and how to compute them. It is a different matter to know when to use which statistic. Knowledge-base theorists, such as Newell and Simon (1972) and Anderson (1987), have provided important insights into the need to help students conditionalize their knowledge so that they can acquire knowledge in the form of condition-action pairs rather than isolated facts.

The results of the present study and those of the previous experiments conducted by Perfetto et al. (1983) make sense from the perspective outlined by Simon (1980). Consider, first, the productions that would be assumed to be generated from exposure to our fact-oriented acquisition sentences. Subjects' processing of these statements might have resulted in condition-action pairs that were minimally helpful for subsequent problem solving given the constraints of the task. Our acquisition task involved the goal of specifying the general truthfulness of statements such as "Before it is played, the score of any game is 0 to 0 ." Given this goal, a general action is to retrieve information about games from memory and check to see if they all begin with no score. This type of conditionaction pairing is very different from what is needed to solve the superpsychic problem under conditions of being uninformed. In contrast, for the informed problem solving condition, the instruction specifies the goal of using what was just learned to solve the problems. Under these conditions, subjects can use well-learned memory productions first to recreate their initial learning context and then to find the answers that are relevant to each problem.

We hypothesize that our problem-oriented acquisition helped students generate condition-action pairs that were much more appropriate for uninformed problem solving. For example, our subjects who received problem-oriented acquisition may have generated productions such as "Given the goal of predicting the score of any game, check to see whether the problem involves the initial score rather than the final score"; or "Given the goal of understanding why it might be commonplace to marry several times per week, check to see if the interpretation of marry can be 'conduct a marriage ceremony' rather than 'get married.' "' If students tended to form these problem-specific productions, this could account for our findings that when fact- versus problem-oriented statements are manipulated as a within-subjects variable, access is facilitated only for those problems whose initial answers appeared in a problem solving format.

Further work is necessary to determine whether existing theories of production systems can adequately account for findings like those obtained in the present experiments. Nonetheless, both the present work and the original work of Perfetto et al. (1983) demonstrate the importance of processes that are relatively knowledge specific. Both the present item-specific positive transfer effect and the previous (replicated) interference effects seem to be tied to specific items. There is little evidence for either positive transfer effects due to general sets or for general interference (negative transfer) effects.

\section{REFERENCES}

ANDERSON, J. R. (1987). Skill acquisition: Compilation of weak-method problem solutions. Psychological Review, 94, 192-210.

AsCH, S. E. (1969). A reformulation of the problem of associations. American Psychologist, 24, 92-102.

Bransford, J. D., Franks, J. J., Morre, C. D., \& Stein, B. S. (1979). Some general constraints on learning and memory research. In L. S. Cermak \& F. I. M. Craik (Eds.), Levels of processing and human memory. Hillsdale, NJ: Erlbaum.

Bransford, J. D., Franks, J. J., Vye, N., \& SHerwood, R. (1986, June). New approaches to instruction: Because wisdom can't be told. Paper presented at the Illinois Conference on Similarity and Analogy, Champaign-Urbana, IL.

Bransford, J. D., \& Johnson, M. K. (1972). Contextual prerequisites for understanding: Some investigations of comprehension and recall. Journal of Verbal Learning \& Verbal Behavior, 11, 717-726.

Bransford, J. D., McCarrell, N. S., FranKs, J. J., \& Nitsch, K. E. (1977). Toward unexplaining memory. In R. E. Shaw \& J. D. Bransford (Eds.), Perceiving, acting, and knowing: Toward an ecological psychology (pp. 431-466). Hillsdale, NJ: Erlbaum.

Bransford, J. D., Sherwood, R., Vye, N., \& Rieser, J. (1986). Teaching thinking and problem solving: Research foundations. American Psychologist.

Chase, W. G., Simon, H. A. (1973). The mind's eye in chess. In W. Chase (Ed.), Visual information processing (pp. 215-281). New York: Academic Press

Craik, F. I. M., \& Tulving, E. (1975). Depth of processing and the retention of words in episodic memory. Joumal of Experimental Psychology: General, 104, 268-294. 
DEGroot, A. D. (1965). Thought and choice in chess. The Hague: Mouton.

DUNCKer, K. (1945). On problem-solving. Psychological Monographs, 58(270).

Franks, J. J., Bransford, J. D., Auble, P. M. (1982). The activation and utilization of knowledge. In C. R. Puff (Ed.), Handbook of research methods in human memory and cognition. New York: Academic Press.

Gick, M. L., Holyoak, K. J. (1980). Analogical problem solving. Cognitive Psychology, 12, 306-355.

Gick, M. L., \& HoLYOAK, K. J. (1983). Schema induction and analogical transfer. Cognitive Psychology, 15, 1-38.

Morris, C. D., Bransford, J. D., \& Franks, J. J. (1977). Levels of processing versus transfer appropriate processing. Journal of Verbal Learning \& Verbal Behavior, 16, 519-533.

NewEll, A., \& SimON, H. A. (1972). Human problem solving. Englewood Cliffs, NJ: Prentice-Hall.

Perfetto, G. A., Bransford, J. D., \& Franks, J. J. (1983). Constraints on access in a problem solving context. Memory \& Cognition, 11, 24-31.

ReEd, S. K., ERnst, G. W., \& Baneru, R. (1974). The role of analogy in transfer between similar problem states. Cognitive Psychology, 6, 436-450.

Simon, H. A. (1980). Problem solving and education. In D. T. Tuma \& R. Reif (Eds.), Problem solving and education: Issues in teaching and research (pp. 81-94). Hillsdale, NJ: Erlbaum.

Simon, H. A., \& HAYES, J. R. (1976). The understanding process: Problem isomorphs. Cognitive Psychology, 8, 165-190.

Weisberg, R., DiCamillo, M., Phillips, D. (1978). Transferting old associations to new situations: A nonautomatic process. Journal of Verbal Learning \& Verbal Behavior, 17, 219-228.

\section{APPENDIX}

Below are the problems and their acquisition sentence clues used in the present snudy. The first sentence below each problem is the sentence used for the fact-oriented conditions. The second sentence below each problem is the sentence used for the problem-oriented pause and no-pause conditions.

One day a lady in New York City hailed a passing taxicab. On the way to her destination, the lady talked so much that the driver got quite annoyed.

The driver finally said, "I'm sorry, lady, but I can't hear a word you're saying. I'm deaf as a post, and my hearing aid hasn't worked all day."

When she heard this, the lady stopped yakking. But after she left the cab, she suddenly realized that the cabbie had lied to her. How did she know he had lied?

1. A cab driver must be told your destination before he takes you there.

2. A cab driver cannot take you anywhere; unless you tell him your destination.

Can you make a tennis ball go a short distance, come to a dead stop, then reverse itself, and go in the opposite direction?

Note: Bouncing the ball is not permitted, nor can you hit it with anything, nor tie anything to it.

1. If you throw a ball into the air, it comes back down.

2. A ball will always return to you; if you throw it up in the air.

A man who lived in a small town in the U.S. married twenty different women of the same town. All are still living and he has never divorced one of them. Yet he has broken no law. Can you explain?

1. A minister marries several people each week.

2. You can marry several people each week; if you are a minister.

Whenever my aunt comes to visit me at the apartment, she always gets off the elevator five floors beneath my floor. She then walks up the stairway to my apartment. Can you tell me why?

1. The top buttons on an elevator are too high for a midget to reach.

2. Some adults cannot reach the top buttons on an elevator; because they are midgets.

John was driving to Las Vegas for a vacation when his car broke down in a small town. While the car was being fixed, John decided to get a haircut. The town had just two barber shops, Joe's and Bill's. John looked through the window of Bill's shop and was disgusted.

"What a dirty shop," he said. "The mirror needs cleaning; there's hair all over the floor; the barber needs a shave, and he has a terrible haircut." It's no wonder that John left Bill's shop and went up the street to check on Joe's Barber Shop. John peeked though Joe's window. "What a difference!" John sighed.

The mirror was clean, the floors were clean, and Joe's hair was neatly trimmed. But John didn't go in. Instead, he walked back to get his hair cut at Bill's dirty shop. Why?

1. A hairdresser will go to another hairdresser to get their hair done.

2. A great hairdresser can have a terrible hairdo; if someone else does their hair.

"This myna bird," said the pet shop salesman, "will repeat anything it hears." A week later the lady who bought the bird was back in the shop to complain that she had talked to the bird, but he had not yet said anything. Nevertheless, the salesman told the truth. Explain!

1. A deaf parrot will not learn to mimic sounds.

2. A parrot will not learn to mimic sounds; if it is deaf.

One night my uncle was reading an exciting book when his wife turned out the light. Even though the room was pitch dark, he continued to read. How could he do that?

1. A blind person can read braille in the dark.

2. It is possible to read in the dark; if you are reading braille.

Uriah Fuller, the famous superpsychic, can tell you the score of any baseball game before the game starts. What is his secret?

1. Before any game is played, there is no score.

2. You can tell the score of any game before it is played; because there is no score.

Last week I turned off the light in my bedroom and managed to get to bed before the room was dark. If the bed was ten feet from the light switch, how did I manage to get to bed while there was still light? 
1. If it's daytime, turning off the light will not make the room darker.

2. Turning off the light will not make the room darker; if it is daytime.

The Reverend Sol Loony announced that on a certain day, at a certain time, he would perform a great miracle. He would walk for twenty minutes on the surface of the Hudson River without sinking into the water. A big crowd gathered to witness the event. The Reverend Sol Loony did exactly what he said he would do. How did he manage to walk on the surface of the river without sinking?

1. A person walking on frozen water will not fall through.

2. A person walking on water will not fall through; if it is frozen.

(Manuscript received October 2, 1986; revision accepted for publication August 25, 1987.) 